\title{
A Case for Combinatorics: A Research Commentary
}

\section{Elise Lockwood Oregon State University}

\author{
Nicholas H. Wasserman \\ Teachers College
}

\section{Erik S. Tillema Indiana University}

\begin{abstract}
In this commentary, we aim to make a case for the explicit inclusion of combinatorial topics in the curriculum - both in K-12 classrooms and in introductory postsecondary mathematics courses - where it is currently essentially absent. To do so, we suggest ways in which researchers might inform the field's understanding of combinatorics and its potential role in curricula. We reflect on five decades of research that has been conducted since a call by Kapur (1970) for a greater focus on combinatorics in mathematics curricula. We offer five assertions about combinatorics, including three existing assertions and two new assertions that relate to increasingly relevant trends in mathematics education. Specifically, we discuss the following in making our case for combinatorics: 1) Combinatorics is accessible, 2) Combinatorics problems provide opportunities for rich mathematical thinking, 3) Combinatorics fosters desirable mathematical practices, 4) Combinatorics can contribute positively to issues of equity in mathematics education, and 5) Combinatorics is a natural domain in which to examine (and develop) computational thinking and activity. As we discuss each of these ideas, we summarize and synthesize existing research and offer new ideas for research. Ultimately, we hope to make a case for the valuable and unique ways in which combinatorics might effectively be leveraged within K-16 curricula, and we hope to elevate its status in the mathematics education research community.
\end{abstract}

This is the author's manuscript of the article published in final edited form as:

Lockwood, E., Wasserman, N. H., \& Tillema, E. S. (2020). A case for combinatorics: A research commentary. The Journal of Mathematical Behavior, 59, 100783. https://doi.org/10.1016/j.jmathb.2020.100783 


\section{A Case for Combinatorics: A Research Commentary}

\section{Introduction and Motivation}

Although there have been recent exceptions, the K-16 mathematics curriculum has remained largely unchanged for decades. Calculus, an important area of mathematics developed in the $17^{\text {th }}$ century, remains the focal point of much of student's requisite mathematical learning, driving the algebra-heavy curriculum of middle and secondary schools. A common course sequence for high school students to take is a year studying each of Algebra, Geometry, Algebra II, and Trigonometry/Pre-Calculus. Given this course sequence, it would not be uncommon, or untrue, for such students to conclude that algebra is mathematics. Yet while the world has changed, perhaps most drastically in the realm of computing and technology, course sequences in mathematics have remained the same. We see this as problematic and detrimental for several reasons that we elaborate in this paper.

The goal of this commentary is to make a case for combinatorics. That is, we aim to make a case for the explicit inclusion of combinatorial topics in the curriculum - both in K-12 classrooms and in introductory postsecondary mathematics courses - where it is currently essentially absent. We regard such inclusion as beneficial both to students and to society. For students, this inclusion both provides accessible opportunities for inclusion around mathematical learning, and strengthens their mathematical development by deepening their mathematical thinking and expanding their notions of mathematics. For society, this inclusion offers opportunities to improve civic engagement in a technological world.

As a research commentary, we specifically look at how existing research has, and future research could, draw the field's attention to the importance of combinatorics and its potential as a key element of K-16 curricula. We frame the commentary by focusing on five assertions that 
emphasize the importance of combinatorics in mathematics education. Specifically, we discuss each of these assertions in turn: 1) Combinatorics is accessible, 2) Combinatorics problems provide opportunities for rich mathematical thinking, 3) Combinatorics fosters desirable mathematical practices, 4) Combinatorics can contribute positively to issues of equity in mathematics education, and 5) Combinatorics is a natural domain in which to examine (and develop) computational thinking and activity. Our goal is both to summarize existing research in combinatorics education to date (particularly as such work addresses each claim above), and to highlight areas in which additional research is needed to provide further evidence to substantiate these claims. In this way, we can both articulate the current state of research in combinatorics education and provide an agenda for future research. Three of these assertions are rationales based in existing reasons and calls related to combinatorics (in particular Kapur's (1970) appeal for more research into combinatorics education nearly fifty years ago), and we believe that these rationales are still relevant today. In discussing them, we demonstrate ways in which researchers have attempted to answer those calls in the intervening fifty years by summarizing existing research, and we highlight potential places where researchers could continue to make inroads in substantiating these particular claims by suggesting avenues for future research. Then, we highlight two new arguments for the importance of combinatorics in mathematics education that previously have not been emphasized explicitly, but that align with two trends in mathematics education research today. By highlighting these new rationales, we argue that we are making a timely case for combinatorics education and research, offering both a retrospective view of what has been done in the fifty years since Kapur's paper, and proposing a forward-looking agenda for what research might continue to support the case that combinatorics deserves a substantive place in $\mathrm{K}-16$ mathematics education. 
Before we articulate the problem that we are trying to address, we first briefly specify what we mean by combinatorics in this paper. ${ }^{1}$ Combinatorics is a topic that sometimes faces definitional confusion (Berge, 1971), and we adopt the following characterization of the term combinatorics. In his Introductory Combinatorics textbook, Brualdi defines combinatorics in the following way: "Combinatorics is concerned with arrangements of the objects of a set into patterns satisfying specified rules...Thus, a general description of combinatorics might be that combinatorics is concerned with the existence, enumeration, analysis, and optimization of discrete structures" (Brualdi, p. 1-2, emphasis in original; note here discrete here means noncontinuous). Others offer similar definitions (see, e.g., Cameron, 1994; English, 2005; Mazur, 2010; Tucker, 2002), all of which capture the idea that combinatorics tends to involve discrete, finite sets or structures, answering questions about the existence, enumeration, and properties of such structures.

\section{Context and Motivation - A Brief History of Combinatorics in the Curriculum in the U.S.}

We first note we are writing from the perspective of researchers in the United States where the problem we are trying to articulate is especially acute. However, we acknowledge that in some countries, including Germany (Höveler, 2017), Israel (Eizenberg \& Zaslavsky, 2003, 2004), Brazil (Borba, Pessoa, Barreto, \& Lima, 2011), Spain (Batanero, Navarro-Pelayo, \& Godino, 1997; Batanero, Godino, \& Navarro-Pelayo, 2005; Godino, Batanero, \& Roa, 2005), and Hungary (Vanscó, Beregszászi, Burian, Emese, Stettner, \& Szitányi, 2016), to name a few, combinatorics already has a strong presence in school curricula. We do not have space to address

\footnotetext{
${ }^{1}$ We note that many of the arguments we are making could also be applied to discrete mathematics more broadly; however, we focus on combinatorics both because, as combinatorics education researchers, we are most qualified to speak more specifically about combinatorics, and because we hope a narrower focus will help to sharpen our arguments.
} 
how combinatorics is treated in each of these other countries, but we use the fact that many other countries do include combinatorial topics as motivation to enact change in the U.S. We now briefly elaborate on the status and history of combinatorial topics in mathematics education in the U.S., which is part of our motivation for writing this commentary.

Combinatorics is a subset of discrete mathematics, and so some discussion of combinatorics necessarily involves treatment of discrete mathematics more broadly. In early standards documents, NCTM (1989) included discrete mathematics (in addition to algebra, functions, geometry, trigonometry, probability, statistics, pre-calculus) as an explicit content area to be incorporated across the high school curriculum, and NCTM offered resources to support this (e.g., NCTM, 1991). This was relatively forward-thinking; it was based on NCTM's (1980) call for action that explicitly made a goal of having a "flexible curriculum, with a greater range of options...to accommodate the diverse needs of the student population" (p. 17). Indeed, the specific inclusion of discrete mathematics (including combinatorics) in the 1989 standards might have even been in response to Kapur's calls in the 1970s, or to other societal developments such as handheld computing devices like calculators and personal computers. Some states, such as New Jersey, made significant headway into having discrete mathematics standards in schools (Rosenstein et al., 1997).

Yet in the decades that have followed, discrete mathematics has slowly been removed from the K-12 curriculum, and it continues to be on the periphery in undergraduate mathematics. Only a decade after the 1989 standards, in NCTM's (2000) standards document, discrete mathematics was no longer an explicit content area; there was only one mention of a combinatorial topic in the Grade 9-12 standards for Number and Operation: "develop an understanding of permutations and combinations as counting techniques." There were some combinatorial topics that were 
obliquely included with the data and probability strand (e.g., computing probabilities, sample space, etc.), but incorporating combinatorial topics solely within probability is not clearly representative of including a combinatorial topic within the curricula. The more recent Common Core State Standards for Mathematics (National Governors Association Center for Best Practices, 2010) implemented in a majority of states in the U.S., further separate discrete mathematics from schools. There are two standards that make explicit mention of combinatorial ideas - one in relation to algebra and the binomial theorem, the other in relation to probability; however, both of these are (+) standards, which means they are optional - i.e., only for those intending further mathematical study. In a concrete sense, combinatorics has been relegated out of the K-12 curriculum in the U.S. - it is not framed as something that students should study as part of their mathematical education. We interpret this development to be a move backward, particularly given how society is changing technologically; a narrowing and standardization of mathematics, rather than an injection of diversity and flexibility into the curriculum. Furthermore, at the college level, although students have more opportunities to engage with combinatorial topics, typically these arise either as a small unit in a transition-to-proof discrete mathematics class, or, perhaps, as an elective course for mathematics majors. That is, combinatorics again generally appears to be viewed as an unimportant aspect of mathematical study.

Now, one might argue these developments happened for good reason, and society learned a lesson and changed course after having attempted to incorporate discrete mathematics into schools in the 1990's. We, however, believe these developments have been a misstep. Somehow over the years, the case for combinatorics became lost, and educators and policymakers forgot 
(or never internalized) the promise for how beneficial combinatorics could be for students. With this commentary, we aim to formulate and elaborate this case for combinatorics.

\section{Renewing assertions about the nature of combinatorics in mathematics education}

We acknowledge that there have been individuals, and groups, who have made a general case for discrete mathematics (and, to some extent, combinatorics) in the past. We seek to build on such prior arguments. In particular, we take time here to elaborate and explore some of these previous rationales, attempting to defend why we have something novel to contribute to the conversation. We first note that in 1970, Kapur published an article in Educational Studies in Mathematics, entitled "Combinatorial analysis and school mathematics," which argued for further inclusion of combinatorics into the curriculum even fifty years ago. We provide his list of 11 reasons in Figure 1. 
Combinatorial mathematics is an essential component of the mathematics of the discrete and as such it has an important role to play in school mathematics. This role has been little exploited so far.

Some of the reasons which make combinatorial analysis important in school mathematics are the following:

(a) Since it does not depend on calculus, its problems can be taken up at an early stage in the school curriculum. In fact it has problems suitable for all grades.

(b) It can be used to train students in the concepts of enumeration (counting with counting through counting without counting), making conjectures, generalizations, optimization, existence, systematic thinking etc.

(c) Applications to physics, chemistry, biology, network analysis, design of experiments, communication theory, symmetry, probability, dynamic programming, number theory, topology, recreational mathematics etc. can be indicated.

(d) The need for creation of more mathematics can be created in the minds of the students. A large number of challenging problems can be indicated to them.

(e) Distinction between plausible and rigorous proofs can be brought out.

(f) Enough motivation for working with computers can be provided.

(g) Students can appreciate the powers and limitations of mathematics as well as the powers and limitations of computers through combinatorial mathematics.

(h) It can help in the development of concepts of mapping, relations, functions, equivalence relations, equivalence classes, isomorphisms etc. rather clearly.

(i) Some of the great victories of the human mind over challenging problems can be indicated.

(j) Most of the combinatorial problems and their applications have been developed recently and so students can get a feeling for the growth of mathematics.

(k) This can help in developing the combinatorial attitude of mind which examines all possibilities, enumerates them and finds out the best possibility and thus leads to clearheaded thinking.

Figure 1: Kapur listed reasons for why combinatorial analysis are important for school mathematics (Kapur, 1970, p. 114).

Kapur did not offer empirical evidence for these claims, and this was not his goal. Rather, he sought to argue his case and to suggest that the field take up the mantle of exploring and

substantiating his claims via research. He presented a number of combinatorial problems that

illustrated his points, and he said the following in his conclusion:

It will require a great deal of investigation, research, and experimentation to find to which problems of combinatorial mathematics can go to school, but it is obvious that along with algebraic structures and geometric transformations, combinatorial problems provide a rich storehouse for modernizing and revitalizing our school curriculum. It is the author's hope that this source will be fully exploited in the future (Kapur, p. 127). 
Now, nearly fifty years later, we can examine how combinatorics education has advanced and how Kapur's calls have been addressed.

Notably, in the intervening there have been other efforts to address the need for more discrete mathematics in the curriculum, most of which involved broad discussions of discrete mathematics (rather than combinatorics specifically). These included, for example, the work of Rosenstein et al. and the DIMACS group about discrete mathematics in schools (e.g., DeBellis \& Rosenstein, 2004; Rosenstein et al., 1997) and the Topic Study Group (TSG) 17 from ICME 2016, which focused on the teaching and learning of discrete mathematics and yielded a book that argued for the value of discrete mathematics (e.g., Hart \& Sandefur, 2017). Others have been more specific to combinatorics. English (2005), for example, highlighted a 1986 Working Group (K-4) of the Commission on Standards for School Mathematics that recommended combinatorics, in elementary school, as "an area of exploration within two of its themes for curriculum development” (p. 121); she also argued that much of Kenney and Hirsch's (1991) Discrete Mathematics across the Curriculum, K-12 (Kenney \& Hirsch) focused on "the teaching of combinatorics especially in the middle and secondary school years" (2005, p. 122). While these publications did not typically entail research studies, these chapters offered instructional ideas and motivations for incorporating combinatorics practically into the classroom (e.g., Althoen, Brown, \& Bumcrot, 1991; DeGuire, 1991; Dossey, 1991; Evered \& Schroeder, 1991; Miller, 1991; Schielack, 1991; Spangler, 1991). In addition, Maher and incorporated combinatorics as a key content area in their longitudinal study out of Rutgers University, and their work culminated in a book entitled Combinatorics and reasoning: Representing, justifying and building isomorphisms (Maher, Powell, \& Uptegrove, 2011) that highlights particularly important aspects of combinatorics among school-age children, and other teachers and 
researchers have made arguments for the importance of combinatorics over the years (e.g., Batanero et al., 2005; Hurdle, Warshauer, \& White, 2016; Sriraman \& English, 2004).

\section{Assertions about Combinatorics: Findings from Research and Opportunities for Additional Investigation}

In this section, we highlight five assertions about why, and in what ways, combinatorics might have value in school mathematics. For each assertion we will summarize and exemplify existing literature related to that assertion, and in this way, this section also offers an extensive literature review of research on combinatorics education. Then, we will also suggest ideas for what additional research might be needed to better understand and substantiate each assertion systematically. Our aim is not merely to state the problem, but to present some practical ideas for the kinds of research studies that might help make a case for (and ultimately enact change in terms of) incorporating combinatorics into K-16 curricula.

\subsection{Combinatorial Tasks Are Accessible and Require Little Mathematical Background Knowledge}

One assertion is that combinatorics, as a mathematical domain, is accessible. By accessible, we mean that combinatorics problems are characterized by: i) asking concrete questions with little technical terminology, (i.e., what the question is asking is easy to understand, with solutions often being concrete counting numbers that answer the question "how many"); and ii) requiring few mathematical prerequisites for a student to have a chance to explore solutions to those problems. Accessibility is an important consideration for thinking about education and school mathematics, and the more accessible the material, the fewer barriers there are to having students conceptually understand and engage in solving mathematical problems. An implication of combinatorics being accessible is that even very young children, as well as students who have not had much success with algebraic (or other mathematical) topics, have a chance to 
meaningfully understand and engage with interesting problems. We discuss how combinatorics education researchers have implicitly supported this assertion. We then suggest some ideas for research studies that could address this claim more explicitly.

Researchers have implicitly highlighted the accessibility of combinatorics by demonstrating student reasoning about counting problems in their studies. In studies about combinatorial learning (e.g., Batanero, et al., 1997; English, 1991, 1993; Eizenberg \& Zaslavsky, 2004; Fischbein \& Gazit, 1988; Fischbein \& Grossman, 1997; Fischbein, Pampu, \& Manzat, 1970; Lockwood \& Gibson, 2015; Lockwood \& Purdy, 2019a, 2019b; Lockwood, Wasserman, \& McGuffey, 2018; Maher \& Martino, 1996a; Maher \& Martino, 1996b; Maher, et al., 2011; Tillema, 2013, 2014, 2018, in press; Tillema \& Gatza, 2016), counting problems often arise from every day contexts. This tends to be true of the field of combinatorics in general, but it is certainly true of the set of problems explored in such research. For instance, consider a problem like the Four-Topping Pizza Problem discussed by Maher, Sran, \& Yankelewitz (2011), and at length throughout Maher, et al. (2011):

Kenilworth Pizza has asked us to help design a form to keep track of certain pizza choices. They offer a cheese pizza with tomato sauce. A customer can then select from the following toppings: peppers, sausage, mushrooms, and pepperoni. How many choices for pizza does a customer have? List all the possible choices. Find a way to convince each other that you have accounted for all possibilities (Maher, Sran, \& Yankelewitz, 2011, p. 69).

The students were in fifth grade when they solved this problem. In reading this problem, we suggest two things. First, it is not difficult to understand what the question is asking. Ordering pizzas is a real world, concrete idea, something children may be familiar with. Further, there is no "mathematical terminology" that needs to be unpacked in the question. Second, even if students do not know how to solve the problem (or could not articulate a formula or general approach), they often can begin to symbolize the set of outcomes (e.g., a sequence Pi, M, S 
would indicate a pizza had pepperoni, mushrooms, and sausage). This is in fact what Maher et al. document, that students represented and listed outcomes, ultimately engaging in meaningful justification to explain their work.

At the elementary level, researchers have shown that even very young children are able to successfully symbolize sets of outcomes with the aid of concrete materials (English, 1991, 1993; Maher, Sran, Yankelwitz, 2010; Nunes \& Bryant, 1996). At the middle grades or high school levels researchers have demonstrated that students can symbolize the set of outcomes using written lists (Speiser, 2011, Tillema, 2013), tree diagrams (e.g., Fischbein \& Gazit, 1988), or arrays (Tillema, 2018). Researchers have demonstrated how symbolizing the set of outcomes can lead to productive and important conversations about fundamental aspects of problems like whether to include ordered outcomes (Tillema, in press) or how to organize a list to determine whether it contains all possible outcomes (Muter \& Uptegrove, 2011). Even with partial listing, students with no prior combinatorial experience can identify patterns and structure within partial lists of outcomes (Lockwood \& Gibson, 2016). This is what we mean when we say that a counting problem is accessible — students are able to understand what a question is asking, they can begin a solution path by symbolizing outcomes, and in the process of symbolizing these outcomes they can have discussions of key combinatorial ideas.

This kind of accessibility stands in contrast with many other problems in school mathematics. For example, problems like the following may not be inherently tricky or difficult, but they require certain background knowledge and expertise: "Prove the identity $\sin ^{2}(x)+\cos ^{2}(x)=1$," "Determine the roots of the following polynomial," "Find a function that is differentiable on $\mathbf{R}$ except at $x=0$," and "Is the following topological subspace homeomorphic?" For these problems, it would be necessary to unpack certain terms and concepts (e.g., what is a "root") before one 
could even parse the problem statements. That is, common questions in many other mathematical areas often have specific, technical language that needs to be defined in order for one to understand the problem prior to attempting to solve it.

Researchers have implicitly shown that combinatorial problems are accessible. However, as part of an argument for incorporating combinatorial problems (as opposed to other kinds of mathematical problems) into K-16 mathematics, there is still a need for clearer empirical demonstrations about the accessibility of combinatorial problems. We elaborate on a few possible ideas for studies that could not only demonstrate the accessibility of combinatorics problems, but that could further show that this is actually beneficial for students.

First, as a field we might simply explore the use of technical language in combinatorial problems versus algebra or geometry problems. For instance, we might analyze prerequisite mathematical knowledge required to solve problems: either exploring how many mathematical prerequisites there are for a typical problem, or how elementary those prerequisites are (e.g., a prerequisite of solving equations would be more advanced (less elementary) than one of addition). Either way, by analyzing problems in textbooks, the field might be able to further interrogate the claim that combinatorial problems are "more accessible" than other content areas, in terms of less technical language, or in terms of fewer, more elementary, mathematical prerequisites. We do not currently actually have measures of accessibility, but such information could support the claim that combinatorial problems are "more" accessible than other domains. Second, we might explore this claim of accessibility empirically with students. Through interviews, one might probe students' understanding of different problems in terms of what problems are asking, to see whether combinatorics, as a field, engages students in solving problems that are "more accessible" than other areas. That is, accessibility is fundamentally 
mediated by the interpreter, and exploring students' interpretations (including different ages, groups, levels of mathematical experience, etc.) would further provide a sense of whether "accessibility" is a characteristic relatively unique to combinatorics as compared to other mathematical areas. If such evidence was found, it could bolster the rationale for incorporating these particular kinds of problems into school mathematics. Third, introductory content and ideas, as opposed to those that are building on those introductory foundations, tend to require less technical vocabulary. It may be that the "accessibility" argument about combinatorics is really an observation related to the level of introduction. Because we study algebra for years, for instance, algebra problems naturally use more technical vocabulary, as such problems are continuing to build on introductory ideas. Combinatorial problems, on the other hand, may tend to be introductory simply because they are not built upon to the same extent that algebra problems are, and so they may have less technical language. However, we would argue that, regardless of whether this is the case or not, there seems to be more one can do with introductory ideas in combinatorics than in other fields; that is, other fields necessitate building on concepts more quickly than one needs to in combinatorics. Parsing the introductory nature of problems versus the mathematical domain would be a challenging but interesting research endeavor and could provide further empirical evidence that could be used to inform the accessibility of combinatorial problems.

The discussion around accessibility raises two issues, which we address briefly. The first is that accessibility does not mean problems are trivial, a notion we elaborate further in the next assertion (Section 4.2) about combinatorial problems providing sufficiently challenging, rich mathematical thinking. The second is related to issues around equity. The fact that combinatorics lacks prerequisites not only makes it so that more people can understand problems, but it also 
opens access for broader populations of people to engage meaningfully with mathematical ideas and topics. In this way, we view combinatorics as a topic that can be used to contribute positively to issues of equity and access that the field of mathematics education is currently considering. This is a topic we will discuss further in Section 4.4.

\subsection{Combinatorics Problems Provide Opportunities for Challenging, Rich Mathematical Thinking for All Students}

In noting that combinatorial problems are accessible, we do not want to imply that they are easy or trivial for students to solve. Indeed, a number of researchers have documented the difficulties that students of all ages face in solving such problems (e.g., Annin \& Lai, 2010; Batanero et al., 1997; Eizenberg \& Zaslavsky, 2004; Hadar \& Hadass, 2004; Lockwood \& Gibson, 2016). ${ }^{2}$ Yet, these challenges do not mean that combinatorial problems are inaccessible or too difficult for students. Rather, we regard these difficulties as suggesting that although combinatorial problems can be easy to engage with because their statements are easy to understand, they also require careful thought and certain practices to master or to understand completely. This means that such problems can appeal to a wide swath of students because they are generally comprehensible to students while also providing engaging challenges. Kapur (1970) captures this particular feature of combinatorial problems nicely: "Some of these problems look trivial in their statements and they present great difficulties in their complete solutions, but most of these can be discussed at the school level" (p. 126). This sums up our characterization of combinatorial problems as being accessible but still able to pose "great difficulties" (or we might say, engaging challenges) in their complete solutions. We discuss three

\footnotetext{
${ }^{2}$ We do not outline causes for such difficulty in this paper, as that is not our primary goal. Some inherent difficulties are discussed in Tucker (2002) and Martin (2001), and reasons for difficulties have been summarized elsewhere, see, for example, Lockwood and Purdy (2019a).
} 
ways in which combinatorics education researchers have supported this assertion. We then suggest some ideas for research studies that could address this claim more explicitly

First, part of what makes combinatorial problems unique (with the potential to be so rich) is that there is not always a clear path or procedure to solve a given counting problem. We support this, first, from mathematicians (textbook authors) providing commentary on their field. Tucker (2002) says of his introductory chapter on counting "In this chapter we discuss counting problems for which no specific theory exists" (p. 169). Brualdi (2004) says, "The solutions of combinatorial problems can often be obtained using ad hoc arguments, possibly coupled with the use of general theory. One cannot always fall back on applications of formulas or known results" (Brualdi, p. 2-3). Martin (2001) notes that "One of the things that makes elementary counting difficult is that we will encounter very few algorithms. You will have to think. There are few formulas and each problem seems to be different" (p. 1). That is, unlike problems in calculus, say, where there might be a clear procedure for solving a problem, there is not always a clear path or process to a solution. This can make counting problems frustrating, but it also sets them apart as commonly requiring ingenuity, cleverness, and novel approaches.

Second, we look at mathematics educators. Annin and Lai (2010) discussed difficulties in teaching students to count, and they note that, "What we often find challenging about teaching students to count, however, is that most problems do not cleanly fall into one and only one standard category of counting problems. Rather, each problem typically involves different aspects that rely on different techniques" (p. 404). That is, one pedagogical implication is that combinatorial methods cannot be reduced to a procedural application, but they require thinking about each problem. As another example, Lockwood, Wasserman, and McGuffey (2019) found that undergraduate students faced difficulties in determining when appropriately to apply a 
formula for combinations, even in the simplest cases where problems fit clearly into the category of being "combination" problems. They also found that when given other problems that could be solved using a combination formula, students did not recognize that they could apply a formula in those cases. The solution path for students, even between relatively simple equivalent problem types, was unclear; they could solve one type of problem but not the other. These comments, and supporting research studies, highlight perhaps some of what makes combinatorics difficult, yes, but they also emphasize what makes combinatorics so unique and engaging - by their very nature, combinatorial problems offer great opportunities for critical thinking and reasoning.

Third, in response to some of these claims about (and calls for) ways in which combinatorics can foster rich mathematical thinking, the combinatorics education community has demonstrated very thoroughly that students can reason richly and deeply within combinatorics. One way in which they have done this is to demonstrate sophisticated student understanding of particular combinatorial topics, such as the multiplication principle (e.g., Lockwood \& Caughman, 2016; Lockwood, Reed, \& Caughman, 2017; Lockwood \& Purdy, 2019a, 2019b), bijections and isomorphism (e.g., Mamona-Downs \& Downs, 2004; Muter \& Maher, 1998; Powell \& Maher, 2003; Tarlow, 2011), combinations and the binomial theorem (Maher \& Speiser, 1997; Lockwood, Wasserman, \& McGuffey, 2018; Speiser, 2011; Tillema \& Burch, 2020; Wasserman \& Galarza, 2019), combinatorial proof (e.g., Engelke \& CadwalladerOlsker, 2010; Maher \& Martino, 1996a, 1996b; Lockwood, Reed, \& Erickson, in press; Tarlow \& Uptegrove, 2011), and equivalence (Lockwood \& Reed, in press). Take, for instance, the multiplication principle (accessible even to elementary students); it is perhaps the most basic counting principle, and yet, in research studies, undergraduate students wrestled with its use in combinatorial problems, taking several hours over the course of a teaching experiment to articulate subtle nuances in the 
principle (Lockwood \& Purdy, 2019a, 2019b). Across these studies, researchers have provided compelling evidence that students can think deeply about a variety of combinatorial topics, and that, even though content and ideas are accessible (e.g., multiplication), combinatorics provides a domain in which they are also sufficiently rich, and challenging, to promote deep mathematical thinking and reasoning. Much of this evidence is qualitative in nature, with researchers demonstrating these findings through task-based interviews (e.g., English, 1991, 1993; Lockwood \& Erickson, 2017; Tillema, 2018, in press) or teaching experiments (e.g., Lockwood, Swinyard, \& Caughman, 2015; Lockwood \& Purdy, 2019a, 2019b; Tillema, 2013, 2014), some of which span long periods of time (Maher, et al., 2011).

Combinatorics education researchers have shown ways in which students reason richly within the domain of combinatorics, but there is more work to be done to support the integration of such topics into the curriculum. One aspect especially in need of further study are what kinds of classroom and teacher supports are necessary to ensure that such reasoning and rich thinking surface. We hypothesize that there could be a two-pronged approach to such work. On the one hand, teachers themselves need sufficient professional development in the area of combinatorics for them to be able to know how even very simple problems might be used to surface rich and deep thinking. We could investigate teachers' explorations of questions such as, What exactly are the nuances of the multiplication principle? or How might a combinatorial problem, or set of problems, be used to engage students richly in thinking about multiplication? On the other hand, implementing classroom tasks is a challenge. We have essentially argued that combinatorics, as a field, provides many problems that have great potential; however, an important pedagogical question to consider is what precisely are the supports to ensure implementation matches potential in combinatorics. Research should help us understand whether there are domain- 
specific ways, in the area of combinatorics, for helping ensure implementation that supports rich thinking.

A second question to consider further relates to investigating typical instructional approaches used in combinatorics versus in other domains. Much instruction in combinatorics is problembased, and particular counting problems are leveraged to develop and apply important combinatorial methods and practices. Algebra teaching, in contrast, can often follow a much stricter procedural approach to learning algebraic methods. However, this need not be the case, and we see opportunities to investigate the relationship between the content that is being taught and the pedagogical approaches to teaching that content. We think it would be valuable to study whether, for example, combinatorics itself as a domain inherently provides better opportunities for rich and challenging thinking than a domain like algebra, or whether any perceived differences about combinatorics are actually a result of an implicit pedagogical approach that provides such mathematical opportunities. Further research that attempts to differentiate, isolate, and understand this interaction would be helpful to investigate whether combinatorics, a particular content area, has some inherent benefits for rich and challenging thinking as opposed to other domains.

\section{3 - Combinatorics Fosters Desirable Mathematical Practices}

There have also been claims that combinatorics can help to foster desirable practices (e.g., Lockwood \& Reed, 2018; Maher et al., 2011). In their presentation of Standards for Mathematical Practice, the CCSSM says, "The Standards for Mathematical Practice describe varieties of expertise that mathematics educators at all levels should seek to develop in their students" (p. 5)." We follow the CCSSM in characterizing mathematical practices as "rest[ing] on important "processes and proficiencies" with longstanding importance in mathematics 
education" (p. 5). Essentially, these standards focus on broad practices, rather than content, that mathematics students should develop over the course of their education. There has been research within the combinatorics education community that both implicitly and explicitly ties to some of these practices.

For example, one such practice in the CCSSM is, Construct viable arguments and critique the reasoning of others, and there are examples of students engaging in this practice in the context of combinatorial problems within the combinatorics education literature. For instance, Lockwood (2014a) showed instances of students trying to make sense of two potential answers, where they needed to think about a hypothetical (incorrect) solution and make sense of it. There have been a number of studies that involve multiple students (such as paired or small-group teaching experiments), where we see evidence of productive discussions between students as they present, consider, and defend their own and others' arguments (Eizenberg \& Zaslavsky, 2004; Kavousian, 2008; Lockwood, et al., 2015; Lockwood \& Reed 2018; Maher, et al., 2011). Often the social dynamic of having students working together in these qualitative studies affords students opportunities to think and reason about their own arguments and the arguments of other students. Because combinatorial problems cannot often be solved by applying a specific procedure (see previous assertion, Section 4.2), students often approach and solve problems very differently (i.e., not using the same prescribed procedure). This is an important quality of combinatorial problems; it suggests that, perhaps more naturally than in other mathematical domains, students will have to defend their own solutions and critique, or come to understand, someone else's. Indeed, part of the fun in combinatorics is realizing that very different solutions, in fact, are both correct. 
As another example, the CCSSM includes the practice to Look for and make use of structure. There are a number of studies that have highlighted ways in which combinatorics is particularly suited for this kind of work. In introductory enumerative combinatorics, there are natural structural distinctions among types of combinatorial objects that are being counted, and this can be a way to distinguish between fundamental types of counting problems. For example, a difference between permutations and combinations can be understood as two different types of counting problems that count two different types of objects (sequences and sets, respectively). Many researchers (e.g., Lockwood \& De Chenne, 2019; Lockwood et al., 2015; Lockwood et al., 2018; Maher et al., 2011; Powell, 2011; Tarlow, 2011) have highlighted structural commonalities and differences among counting problems, and they have documented students' reasoning about such relationships. In addition, some researchers (most notably Batanero et al., 1997) discuss the effects of implicit combinatorial models of combinatorial problems, in particular highlighting differences between selection, distribution, and partition problems (DuBois, 1984). The existence of such models demonstrates important structural aspects of counting problems and highlight ways in which students may meaningfully distinguish between structures within combinatorics.

Another way that researchers have demonstrated that students look for and make use of structure within the domain of combinatorics is through numerical expressions that reflect a particular counting process (Lockwood, 2013; Lockwood \& Reed, 2018). Burch, AtaidePinheiro, and Tillema (2019) have noted a similar phenomenon with pre-service secondary teachers as the PSTs determined binomial coefficients; namely the PSTs used different counting processes for the same problem and the numerical expressions they produced reflected these different counting processes. Finally, some researchers (e.g., English, 1991, Halani, 2012; Lockwood, 2013; Lockwood \& De Chenne, 2019; Lockwood \& Gibson, 2016) have also 
demonstrated how students might leverage structure of combinatorial objects in developing, refining, and implementing listing strategies.

As a final example of ways in which researchers have examined combinatorics as a domain in which to develop mathematical practices, some researchers have explored justification and generalization within the context of combinatorics (e.g., Lockwood, 2011; Lockwood \& Reed, 2018; Reed \& Lockwood, 2018; Maher et al., 2011). One of the central components of justification in combinatorics is providing an argument that demonstrates that one has counted all of the desired outcomes exactly once. For example, Maher and colleagues (Maher \& Yankelwitz, 2011; Maher, Sran, \& Yankelwitz, 2011) have illustrated that as early as the $2^{\text {nd }}$ and $3^{\text {rd }}$ grade children can begin to wrestle with how to justify that they have produced all possible outcomes (see also, English, 1991, 1993, 1996), and that by the $5^{\text {th }}$ grade some students in their study were able to use case-based arguments to aid in their justifications. At the high school level, Tillema and Gatza (2017) have demonstrated the role that justification plays for students prior to them being able to conclude that two different ways of counting the same set of outcomes are equivalent; namely to conclude equivalence the students needed to first justify that each way of counting counted the desired set of outcomes exactly once. In work focused on generalization, researchers have demonstrated that within combinatorics there are many opportunities for students to generalize beyond just finding numerical or algebraic patterns. Indeed, students have been shown to focus on generalizing structural patterns and relationships like those related to the binomial coefficients (Muter \& Uptegrove, 2011) and the multiplication of binomials (Tillema \& Gatza, 2017). Moreover, Burch et al. (2019) showed that pre-service secondary teachers saw different structures in their lists for the same problem, and that these different structures allowed the pre-service teachers to discuss which of the structures were generalizable. Ellis, Lockwood, 
Tillema, and Moore (2017) have also illustrated that students can establish "relations of similarity across problems or contexts" (p. 680) in the domain of combinatorics, a powerful basis for beginning to make abstractions about when situations might require a particular combinatorial operation.

There is relatively strong qualitative evidence that students in individual interviews, pairs, or small groups can engage in the mathematical practices outlined above. We see several potential areas for further research related to mathematical practices. First, the studies cited above show that students can and do engage in powerful ways with particular mathematical practices. However, the extent to which their engagement in these mathematical practices in a combinatorial setting might support them to engage in similar practices in other mathematical domains has not been a focal point of study. Conversely, the way that these mathematical practices entail domain specific ways of thinking has also not been extensively studied. Both of these kinds of investigations could bolster an understanding about mathematical practices, and the assertion that combinatorics can be leveraged to help develop them in productive ways. Second, there have been relatively few studies that investigate how teachers might learn to support students in these mathematical practices in their classroom instruction. Given that K-12 teachers may have limited experiences with combinatorics, this research needs to include studies that provide information on teacher learning in this domain, studies on how teachers come to see this domain as connected to the curricula that they teach, and studies that investigate how teachers implement and support students to engage with mathematical practices in this domain. Notably, this might expose several different approaches for incorporation at the K-12 level; such as incorporating combinatorial topics within the development of existing courses and ideas, or as a course on its own. At the post-secondary level, classroom studies on how instructors learn to 
support students to engage in these mathematical practices may be slightly easier given that collegiate level combinatorics courses already exist.

\section{4 - Combinatorics Can Contribute Positively to Issues of Equity in Mathematics}

\section{Education}

In this section, given the recent long overdue import given to issues around equity in mathematics education, we frame a new assertion about the importance of combinatorics, namely that combinatorics can contribute positively to issues of equity in mathematics education. We acknowledge that this is related to issues of accessibility, but we argue that this is a distinct rationale, as it involves not just the nature of combinatorial content but the impact of that content on who has access to mathematics within society. In particular, we focus on issues of equity and access for students who may not feel welcome as members of the mathematical community (which overwhelmingly runs along gender and racial lines (Gutiérrez, 2008, Martin, 2015)). The premise is for such students, it is possible that some of their mathematical (non)identification may stem from their not being given access to different mathematical domains, ones which might align more closely to their own mathematical ways of thinking and doing. By expanding students' ideas about what mathematics is, we can expand access to who believes they can do, and who sees values in, mathematics. This is in contrast to current school emphases, which, we believe, mistakenly send the message to many students that they are not "mathematics people." Indeed, because combinatorics problems do not require substantial prerequisites or technical terminology, it is particularly well-suited for addressing this issue. Even if students are "behind" in other areas of mathematics (i.e., they have not done well in algebra), they can get a fresh-start in a different area, one that is rooted in the familiar mathematical notion of counting but that also involves sufficiently challenging and rigorous mathematics. We now outline three more specific 
ways in which combinatorial topics could be particularly related to current issues of equity in mathematics education, and we suggest that additional research might be carried out in each of these areas.

First, counting problems can be naturally applied to issues related to social justice. There are many combinatorics problems that can be used to explore relevant social issues. Gatza (2018) has used combinatorics problems to explore how middle grades students' understand racial bias in jury selection (see also Gatza, Tillema, \& Burch, 2020). In his work, he used combinatorics problems to design an intervention intended to help students move from an individual understanding of racial bias to a structural one. As students modeled quantitative relationships from the combinatorics problems, learned about definitions of race and bias, and about how the judicial system works, they came to appreciate the complexity of making a mathematical model of a situation (e.g., Are racial categories discrete? If so, what are the historical origins of treating them as discrete categories? How have these categories changed over time?). Using mathematics to explore relevant social issues is not unique to combinatorics, but because many counting problems arise from readily accessible contexts there is great promise in using combinatorics for such purposes.

Second, combinatorics could expand students' views of what mathematics is. Students may not regard discrete mathematics as "math," perhaps because of how different it seems from algebra and calculus to which they are accustomed. Again, presenting mathematics as a singularity (algebra) potentially limits students' conception of what mathematics is; pointing out discrete mathematics as "math" can expose students to mathematics as a multiplicity. Further, students may not be used to its connection to every day contexts, and the goals of problem solving often seem different from other branches of mathematics. Even historically, discrete 
mathematics was not necessarily considered mathematics by mathematicians. Euler, who first posed and solved the now-famous Königsburg bridge problem in the 1700s, did not even consider the problem to be "mathematics" because it was utterly different from other areas of mathematics. It is now a quintessential problem in graph theory. We see this difference as an inroad to conversations about the nature and applications of mathematics. For example, in contemporary times combinatorics is at the root of how computing systems work, a discussion that we think has the potential to capture students' imaginations (e.g., when I press an "A" on my keyboard why does the letter appear on my screen?; how is a binary number system involved in satellite communication?). These kinds of opportunities allow students to consider (like Euler did) that something they may not recognize as mathematical is in fact deeply mathematical.

Combinatorics provides opportunities for students to see many additional mathematical topics and ideas that offer different perspectives on the nature of mathematics. We see this both in the actual content, and in typical approaches to problem solving within various domains. That is, combinatorics offers new interesting topics that they may not see in algebra or calculus, such as counting techniques and recurrence relations. But, combinatorics also offers a different way of approaching and solving problems. As we have noted, counting requires ingenuity, and solutions do not follow predictable procedures or algorithms. Even seeing that combinatorics requires a different way of approaching problems could expand students' view of the mathematical process, and students who are not satisfied with applying procedures and calculations may be compelled by what combinatorics as a mathematical domain has to offer.

Consider, for instance, combinatorial proofs. These are not exercises in symbolic manipulation, but rather they entail articulating enumerative arguments about certain sets of objects (see Lockwood, Reed, \& Erickson, in press). They even look different than proofs in 
other domains, as they consist simply of sentences of text and may not contain any symbols at all. This stands in contrast to the kinds of proof and argumentation that many students at an early mathematical level are used to, which primarily involve algebraic manipulation. As another example, consider the relationship between discrete mathematics and computers, a topic we discuss further in Section 4.5. Mathematics related to computers involves not continuous structures, but logic, recurrences, combinatorial arguments, algorithms, and more. These are topics that are fundamentally mathematical and are essential to computers, but, in the focus on continuous math of algebra and calculus, many students never learn that mathematics entails such topics.

Third, if students can gain broader perspectives on the nature of mathematics, then we also contend that combinatorics can broaden opportunities for students to feel like they are capable at mathematics. Simply because of curricular emphases on algebra and calculus that we have mentioned, students may conclude that if they do not excel at algebra, they simply are not mathematics people. We thus may lose people to studying mathematics simply because they do not associate their interests, or talents, with mathematics. Indeed, ask many people if they use mathematics in their jobs, and they will say no-that is, rarely do they do some activity they associate with mathematics, like "solving for $x$ " or "applying the quadratic formula." But if people regarded networks, or logistics operations, as graphs from discrete mathematics, or recognized sets and enumeration as informing their daily technological interactions, they might have a different view about mathematics. In other words, some who identify as non-math people may, in fact, be more likely to identify as math people after realizing that mathematics is not only algebra, by being given opportunities to study a fundamentally different domain of mathematics. To be clear, these questions of what mathematics is and who can do mathematics 
are fundamentally issues of access and equity. Currently, people who are considered (by themselves or others) as non-math people base these evaluations on their own perception of mathematics, one that is skewed by their exposure to mathematics from their own learning.

In sum, by studying a different area of mathematics, students who previously did not identify with mathematics might now identify with mathematics; by providing students with an area where problems are easily accessible despite prior study, students who were poor in some areas might find they excel in a different area; and by incorporating combinatorics problems, students can develop computational and other mathematical ways of thinking that give access to technology-based (or other STEM) careers.

We thus see a great need for researchers to study the potential for discrete mathematics, and combinatorics in particular, to capture the interest of and engage students who might otherwise dismiss mathematics. This simply has not been systematically investigated, and results from such studies could provide insight on the extent to which combinatorics can and should be used to broaden participation in mathematics. For example, aside from Gatza's work, we are not aware of studies that have leveraged combinatorial problems to explore issues of social justice. Thus, we see potential for research studies that might actually examine the effectiveness of combinatorics in this context. We also feel strongly that combinatorics could give students opportunities to broaden their perspective on the nature of mathematics. Yet, there is a need to examine this phenomenon empirically. As an example, the field might examine students' current conceptions of mathematics (as related to algebra and calculus versus discrete mathematics and combinatorics), and investigate ways to expand students' views of mathematics through combinatorics. One might ask students, through surveys and interviews, about their conceptions of mathematics, or about which kinds of problems they associate most with mathematics, before 
and after taking a discrete mathematics course. By doing this for different levels and populations of students, we could see what kinds of perspectives exist for various populations. In addition, we could give students problems from different domains and query the extent to which those various problems reflect what the students believe about the nature of mathematics. Then, with such information, we could investigate ways in which to help students actually broaden and expand their views of mathematics, and we could use combinatorics to do so. This might entail giving students combinatorial tasks and also giving reflective questions that draw their attention to the nature of mathematics. Lastly, explicit investigation about access and equity could be valuable. As an example, one might study people who identify as "non-math" people in order to disentangle whether their identification is based on a singular domain-specific view of mathematics; one might explore the effect taking a discrete mathematics has on mathematical self-efficacy for such students. That is, does studying combinatorics actually shift people's mathematical identities, or their sense of self-efficacy in mathematics? In such cases, it might be important to attempt to explore whether shifts in identity are associated with the content in particular, or with an implicit pedagogical approach to teaching that content? Similarly, one might study whether exposing students to different areas of mathematics, including combinatorics, perhaps alters their future mathematical course-taking. Such studies might pave the way for understanding the way that combinatorics might be used to expand access and equity to mathematics. Although we find each of these compelling for their potential, the mathematics education research community needs to take up this issue to explore whether these ideas in fact have merit.

\section{5 - Combinatorics Is a Natural Domain in which to Examine (and Develop) Computational Thinking and Activity}


Our world is increasingly computer-oriented, and our interactions are regularly mediated by technology. Combinatorics is an essential mathematical topic for computer scientists to know and to understand, but it is also increasingly essential to everyday citizens being able to understand, and critically engage in, our technological world. Even more, increasing numbers of fields are involving computing, and more and more jobs involve computational literacy and fluency. Along with others (e.g., Abramovich \& Pieper, 1996; Buteau \& Muller, 2019; Cetin \& Dubinsky, 2017; diSessa, 2018; Hickmott, Prieto-Rodriguez, \& Holmes, 2018; Lockwood, DeJarnette, \& Thomas, 2019; Papert, 1980; Pei, Weintrop, \& Wilensky, 2018; Sinclair \& Patterson, 2018), we are becoming convinced that it is our responsibility as mathematics educators to help our students develop an understanding of computing and to be able to reason about and use computing as a part of their scientific and mathematical work. There have been calls for more integration of computing into curricula (see, for example, CS for all and increasing number of calls by federal and private funding agencies to focus on CS education (e.g., Blikstein, 2018)). With this increased attention on seeing computing integrated more wholly into the curriculum, we contend that we as a field would do well to look to combinatorics as a natural place to introduce fundamental computing structures and concepts into the mathematics curriculum.

There are inherent connections between discrete mathematics and computers and computer science, as data and information must be discretized for computers to be able to process and work with such data. Even decades ago Kapur (1970) raised some of these connections, noting that in combinatorics "enough motivation for working with computers can be provided" (p. 114) and "Students can appreciate the powers and limitations of mathematics as well as the power and limitations of computers through combinatorial mathematics" (p. 114). These comments were 
from fifty years ago, and we now have much clearer examples of such connections to computers. For example, one point of connection is a common process connection between combinatorics and computing. It is very common in combinatorics to break a problem (or proof) up into several cases (often disjoint) and solve each individually; by doing so in a systematic way, we end up with a solution based on cases. This case-based, systematic, way of reasoning is also useful in computing, as many computational approaches consider the space of possible cases, and design solutions to these different cases as a means of solving the larger problem. Because computing, and discrete mathematics, are fundamentally about finite (and not infinite) processes, cases exist and are a useful and productive approach in both domains. This is but one example of ways in which combinatorial topics and approaches can complement ideas in computer science. While Kapur did not elaborate these ideas or show evidence with data, we now have a chance to identify ways in which research has supported these claims over the last several decades, and to make new arguments for additional research that could support these claims.

As an example of work that is exploring relationships between combinatorics and computational thinking and activity in particular, some researchers have investigated ways in which combinatorial thinking may be well-suited for fostering computational thinking and activity, as well as how computational settings might reinforce and enrich students' reasoning about combinatorics problems (e.g., Fenton \& Dubinsky, 1996; Lockwood \& De Chenne, 2019; De Chenne \& Lockwood, 2020; Lockwood, Valdes-Fernandez, \& De Chenne, 2019). For instance, in their book Introduction to Discrete Mathematics with ISETL, Fenton and Dubinsky (1996) proposed a new programming language (ISETL) that would explicitly help to reinforce topics of discrete mathematics in a computational setting. As another example, Lockwood \& De Chenne (2019) report on two students' exploration of tasks in which they solved counting 
problems in a computational setting of programming involving coding in Python. Lockwood and De Chenne provided evidence "that students were able to reason about, understand, and use nested for loops and particular conditional statements within those for loops to computationally generate lists of outcomes for three main types of problems" (p. 47), and they suggested that "the symbols in the Python code seemed to enrich the students' combinatorial reasoning by affording opportunities for the students to make a connection to the kinds of outcomes they were counting" (p. 47). The students in their study, some of whom were novice counters, expressed that they found the context to be an effective introduction to principles of computing.

As a field, then, we are gaining empirical evidence for ways in which combinatorics can help to enrich students' computational thinking and activity, but we see a need continued work in this area. Specifically, the field could conduct studies that investigate what relationships students see between the structure of counting problems and computing languages, which could help to identify what kinds of counting problems and structures students might use to support the development of computing languages and vice versa. Researchers could also analyze extant curricular materials that identify combinatorics problems that afford opportunities for supporting the development of computational thinking. We also consider design experiment studies that investigate novel curricular interventions aimed at supporting the mutual development of combinatorial reasoning and computational thinking to be an important area for further research. That is, prior to implementation in classrooms we think it is important to have studies that test the affordances and limitations of particular curricular interventions in experimental settings in order to inform subsequent classroom implementation. These studies could answer questions such as: What are the necessary ingredients for a curricular intervention to mutually support combinatorial reasoning and computational thinking? In what ways is each mutually supported? 
How do computing foci versus mathematics foci influence the ways in which these two are mutually supported?

\section{Discussion and Conclusion}

In this commentary, we have attempted to make a case that more attention should be paid to combinatorics within K-16 mathematics curricula. We have argued that combinatorics has potentially unique affordances within the realm of mathematics education. In particular two current trends in mathematics education, including increased attention to issues of access and equity, and the current national push toward computational thinking initiatives in mathematics education, suggest that this case for combinatorics is timely. Notably, through this commentary, we have tried to synthesize existing literature from the past five decades in combinatorics education research, while also identifying an agenda for the kinds of work and research that still needs to be done. To conclude, we offer a vision of what a multi-pronged effort in this area, which involves researchers, practitioners, and policy-makers, might look like.

First, for researchers, we regard the exploration and substantiation of claims to be of utmost importance. That is, as a field, to accomplish the incorporation of discrete mathematics and combinatorics into the curriculum, we need strong foundations on which to make such arguments. While existing research provides some of these foundations, there is more to be done. In addition to small qualitative work, we need larger-scale quantitative studies that help us understand generalizations that can be made. We need to not rely just on compelling theoretical arguments about the utility of discrete mathematics (e.g., that it might expand students' sense of what mathematics is), but continue to push for increasing the number and amount of empirical studies and evidence. Such evidence forms the solid backbone of arguments for broader incorporation. 
Second, for practitioners, we need people willing to engage in the practical work of developing curricular ideas and resources in K-16 mathematics. First, for K-16 mathematics educators, we need people willing to generate curricular resources that are age-appropriate for specific students. Notably, this might involve multiple different approaches to thinking about the incorporation and inclusion of discrete mathematics and combinatorics into the curriculum. We name two, as examples. First, we might consider entire units, or courses, devoted to topics in combinatorics. These might be multi-week units, or even semester long courses in high school, but these kinds of approaches would represent a valuing of combinatorics as mathematics in its own right - similar to units and courses on topics in probability, or statistics. As an alternative, we might consider ways that discrete and combinatorial topics might be incorporated for the purpose of deepening students understanding of current curricular goals, for example, in algebra. One could imagine that exploring counting problems, because they lend themselves to different equivalent expressions might help students attend to (and interpret) the structure of algebraic expressions (e.g., CCSSM (2010) standards HSA.SSEA.1 and A.2). That is, combinatorial problems might be used to help students learn algebraic (or other) topics. In either case, we need practitioners working on practical ways to incorporate discrete mathematics and combinatorics into K-16 curricula. Second, for K-16 mathematics teacher educators, we need to consider the practical necessity of teacher preparation. Existing teachers might not have the mathematical expertise to feel comfortable teaching students combinatorial topics; teacher educators are the ones that must be tasked with the challenge of how to prepare a teacher workforce for such incorporation. This includes developing professional development, rethinking teacher preparation program requirements and courses, etc. Practitioners of mathematics teacher education are a necessary prong for realizing such goals. 
Third, for policy-makers, we need public voices and larger policy mechanisms in place to help support and bolster such efforts. Here, we make an analogy to statistics education. Over the past 20 years, statistics educators have been extremely successful at shaping the mathematics curricula to include more statistics. Not only have they worked to build a research based in statistics education, but they have done an excellent job in helping the public understand the importance and necessity of statistics in today's world; the public now generally believes statistics to be very important. Indeed, many in the public argue now that data and statistics should be at least a, if not the, primary part of student's mathematics education. The statistics education community has also benefitted from other driving forces; the existence of an AP Statistics course, as an alternative to AP Calculus, in high school, for example, has been used as a mechanism to increasingly incorporate statistics throughout the curriculum and to provide alternative mathematical options for study. Hence, from this analogy, we see the need for voices to help make the argument to the public for the importance of discrete mathematics and combinatorics to students' mathematical education. Notably, we believe arguments that leverage the current emphases on computational thinking, and on how combinatorial reasoning can help everyday citizens understand the role and nature of computers and algorithms in their everyday lives (which are increasingly mediated by technological interactions), could be especially compelling. Additionally, promoting the diversity of mathematics, and asking the public to demand an education that sheds light on this mathematical diversity, because such diversity is important to conversations about access and equity, is similarly important. Having voices to help shape public perception is an important prong in such efforts. Similarly, efforts to entrench combinatorics as important aims in public policy and the public infrastructure is important. For example, having people fight for the inclusion of standards that emphasize discrete mathematics 
topics; having public organizations such as the National Council of Teachers of Mathematics (NCTM), or the Mathematical Association of America (MAA), etc., vie for education to portray the diversity of mathematics, including discrete; and perhaps even having organizations like the College Board consider the creation of an AP Discrete Mathematics exam. All of these efforts would be useful toward accomplishing the broader aim for which we have advocated in this commentary. 


\section{References}

Abramovich, S., \& Pieper, A. (1996). Fostering recursive thinking in combinatorics through the use of manipulatives and computer technology. The Mathematics Educator, 7(1), 4-12.

Althoen, S. C., Brown, J. L., \& Bumcrot, R. J. (1991). Graph chasing across the curriculum: Paths, circuits, and applications. In M. J. Kenney \& C. R. Hirsch (Eds.), Discrete Mathematics across the Curriculum, K-12: 1991 Yearbook (pp. 30-43). Reston, VA: National Council of the Teachers of Mathematics.

Annin, S. A., \& Lai, K. S. (2010). Common errors in counting problems. Mathematics Teacher, 103(6), 402-409.

Batanero, C., Navarro-Pelayo, V., \& Godino, J. (1997). Effect of the implicit combinatorial model on combinatorial reasoning in secondary school pupils. Educational Studies in Mathematics, 32, 181-199.

Batanero, C., Godino, J., \& Navarro-Pelayo, V. (2005). Combinatorial reasoning and its assessment. In I. Gal \& J. B. Garfield (Eds.), The assessment challenge in statistics education (pp. 239-252): IOS Press.

Berge, C. (1971). Principles of combinatorics. New York: Academic Press.

Blikstein, P. (2018). Pre-College Computer Science Education: A Survey of the Field. Mountain View, CA: Google LLC. Retrieved from https://goo.gl/gmS1Vm

Borba, R., Pessoa, C., Barreto, F., \& Lima, R.C.G. (2011). Children’s, young people’s and adults' combinatorial reasoning. In Ubuz, B. (Ed.). Proceedings of the 35th Conference of the International Group for the Psychology of Mathematics Education (Vol. 2, 169176). Ankara, Turkey: PME

Brualdi, R. A. (2004). Introductory combinatorics (4 ${ }^{\text {th }}$ ed.). Upper Saddle River, New Jersey: Pearson Prentice Hall.

Burch, L.J.., Pinheiro, Attaide, W. \& Tillema, E.S. (2019). Opportunities for generalizing within pre-service secondary teachers'symbolization of combinatorial tasks. Brief research report at the Forty First Annual Meeting of the International Group for Psychology of Mathematics Education in North America, St. Louis, MO: University of Missouri.

Buteau, C. \& Muller, E. (2017). Assessment in undergraduate programming-based mathematics courses. Digital Experiences in Mathematics Education, 3, 97-114. doi:10.1007/s40751016-0026-4

Cetin, I., \& Dubinsky, E. (2017). Reflective abstraction in computational thinking. Journal of Mathematical Behavior, 47, 70-80. 
Cameron, P. J. (1994). Combinatorics: Topics, techniques, algorithms. Cambridge: Cambridge University Press.

DeBellis, V., \& Rosenstein, J. G. (2004). Discrete mathematics in primary and secondary schools in the United States. ZDM, 36, 77-81.

De Chenne, A. \& Lockwood, E. (In press). Student verification practices for combinatorics problems in a computational environment. To appear in the Proceedings of the 23rd Annual Conference on Research in Undergraduate Mathematics Education.

DeGuire, L. J. (1991). Permutations and combinations: A problem-solving approach for middle school students. In M. J. Kenney \& C. R. Hirsch (Eds.), Discrete mathematics across the curriculum, K-12: 1991 yearbook (pp. 59-66). Reston, VA: National Council of Teachers of Mathematics.

diSessa, A. A. (2018). Computational Literacy and “The Big Picture” Concerning Computers in Mathematics Education. Mathematical Thinking and Learning, 20(1), 3-31. https://doi.org/10.1080/10986065.2018.1403544

Dossey, J. A. (1991). Discrete mathematics: The math for our time. In M. J. Kenney \& C. R. Hirsch (Eds.), Discrete mathematics across the curriculum, K-12: 1991 yearbook (pp. 19). Reston, VA: National Council of Teachers of Mathematics.

Dubois, J. G. (1984). Une systematique des configurations combinatoires simples. Educational Studies in Mathematics, 15(1), 37-57.

Eizenberg, M. M., \& Zaslavsky, O. (2003). Cooperative problem solving in combinatorics: The inter-relations between control processes and successful solutions. Journal of Mathematical Behavior, 22, 389-403.

Eizenberg, M. M., \& Zaslavsky, O. (2004). Students' verification strategies for combinatorial problems. Mathematical Thinking and Learning, 6(1), 15-36.

Ellis, A. B., Tillema, E., Lockwood, E., \& Moore, K. C. (2017). Generalization across domains: The relating-forming-extending generalization framework. In Galindo, E. \& J. Newton (Eds.). Proceedings of the $39^{\text {th }}$ Annual Meeting of the North American Chapter of the Psychology of Mathematics Education (677-684). Indianapolis, IN: Indiana University.

Engelke, N., \& CadwalladerOlsker, T. (2010). Counting Two Ways: The Art of Combinatorial Proof. Proceedings of the 13th Annual Conference on Research in Undergraduate Mathematics Education. Presented at the Special Interest Group of the Mathematical Association of America (SIGMAA) for Research in Undergraduate Mathematics Education. Retrieved from http://sigmaa.maa.org/rume/crume2010/Abstracts2010.htm 
English, L. D. (1991). Young children's combinatorics strategies. Educational Studies in Mathematics, 22, 451-47.

English, L. D. (1993). Children's strategies for solving two- and three-dimensional combinatorial problems. Journal for Research in Mathematics Education, 24(3), 255-273.

English, L. D. (1996). Children's construction of mathematical knowledge in solving novel isomorphic problems in concrete and written form. Journal of Mathematical Behavior, 15(1), 81-112.

English, L. D. (2005). Combinatorics and the development of children's combinatorial reasoning. In G. A. Jones (Ed.), Exploring probability in school: Challenges for teaching and learning (Vol. 40, pp. 121-141): Kluwer Academic Publishers.

Evered, L. J., \& Schroeder, B. (1991). Counting with generating functions. In M. J. Kenney \& C. R. Hirsch (Eds.), Discrete mathematics across the curriculum, K-12: 1991 yearbook (pp. 143-149). Reston, VA: National Council of Teachers of Mathematics.

Fenton, W. \& Dubinsky, E. (1996). Introduction to discrete mathematics with ISETL. New York: Springer-Verlag.

Fischbein, E., \& Gazit, A. (1988). The combinatorial solving capacity in children and adolescents. ZDM, 5, 193-198.

Fischbein, E., \& Grossman, A. (1997). Schemata and intuitions in combinatorial reasoning. Educational Studies in Mathematics, 34, 27-47.

Fischbein, E., Pampu, I., \& Manzat, I. (1970). Effects of age and instruction on combinatory ability in children, British Journal of Educational Psychology, 40, 261-270.

Gatza, A.M. (2018). Investigating racism with mathematics: Racial bias, quadratics, and combinatorics. Paper presentation at Annual Meeting of the National Council of Teachers of Mathematics, Washington, DC.

Gatza, A.M., Tillema, E.S., Burch, L.J. (2020). Achieving "strategic outcomes" for effective teaching: Using discrete mathematics to develop content knowledge, design curriculum, and address issues of equity. Symposium at the American Mathematics Teacher Educators annual conference, Phoenix, AZ.

Godino, J., Batanero, C., \& Roa, R. (2005). An onto-semiotic analysis of combinatorial problems and the solving processes by university students. Educational Studies in Mathematics, 60, 3-36.

Gutiérrez, R. (2008). A “gap-gazing” fetish in mathematics education? Problematizing research on the achievement gap. Journal for Research in Mathematics Education, 39(4), 357364. 
Hadar, N., \& Hadass, R. (1981). The road to solving a combinatorial problem is strewn with pitfalls. Educational Studies in Mathematics, 12(4), 435-443.

Halani, A. (2012). Students' ways of thinking about enumerative combinatorics solution sets: The odometer category. In the Electronic Proceedings for the Fifteenth Special Interest Group of the MAA on Research on Undergraduate Mathematics Education. (pp. 59-68). Portland, OR: Portland State University.

Hart, E. W., \& Sandefur, J. (Eds.). (2017) Teaching and learning discrete mathematics worldwide: Curriculum and research. Cham, Switzerland: Springer International Publishing.

Hickmott, D., Prieto-Rodriguez, E., \& Holmes, K. (2018). A scoping review of studies on computational thinking in K-12 mathematics classrooms. Digital Experiences in Mathematics Education, 4, 48-69. doi:10.1007/s4075-017-0038-8

Höveler, K. (2017). Children’s combinatorial counting strategies and their relationship to conventional mathematical counting principles. In E. W. Hart \& J. Sandefur (Eds.) Teaching and learning discrete mathematics worldwide: Curriculum and research (8192). Cham, Switzerland: Springer International Publishing.

Hurdle, Z., Warshauer, M., \& White, A. (2016). The place and purpose of combinatorics. Mathematics Teacher, 110(3), 216-221.

Kapur, J. N. (1970). Combinatorial analysis and school mathematics. Educational Studies in Mathematics, 3(1), 111-127.

Kavousian, S. (2008) Enquiries into undergraduate students' understanding of combinatorial structures. Unpublished doctoral dissertation, Simon Fraser University - Vancouver, BC.

Kenney, M. J., \& Hirsch, C. R. (Eds.). (1991). Discrete mathematics across the curriculum, K12: 1991 yearbook. Reston, VA: National Council of Teachers of Mathematics.

Lockwood, E. (2011). Student connections among counting problems: An exploration using actor-oriented transfer. Educational Studies in Mathematics, 78(3), 307-322. doi:10.1007/s10649-011-9320-7

Lockwood, E. (2013). A model of students' combinatorial thinking. Journal of Mathematical Behavior, 32, 251-265. doi:10.1016/j.jmathb.2013.02.008

Lockwood, E. (2014a). A set-oriented perspective on solving counting problems. For the Learning of Mathematics, 34(2), 31-37.

Lockwood, E. (2014b). Both answers make sense! Using the set of outcomes to reconcile differing answers in counting problems. Mathematics Teacher, 108(4), 296-301. 
Lockwood, E. (2019). Using a computational context to investigate student reasoning about whether “order matters” in counting problems. In Weinberg, A., Moore-Russo, D., Soto, H., \& Wawro, M. (Eds.) Proceedings of the 22nd Annual Conference on Research in Undergraduate Mathematics Education (pp. 385-392). Oklahoma City, Oklahoma.

Lockwood, E. \& Caughman, J. S. (2016). Set partitions and the multiplication principle. Problems, Resources, and Issues in Mathematics Undergraduate Studies, 26(2), 143-157. doi:10.1080/10511970.2015.1072118.

Lockwood, E. \& De Chenne, A. Using conditional statements in Python to reason about sets of outcomes in combinatorial problems. Accepted with revision. International Journal of Research in Undergraduate Mathematics Education.

Lockwood, E., De Chenne, A., \& Valdes-Fernandez, S. (2019). Affordances of solving counting problems in a computational environment. In Graven, M., Venkat, H., Essien, A. \& Vale, P. (Eds). (2019). Proceedings of the $43^{\text {rd }}$ Conference of the International Group for the Psychology of Mathematics Education (Vol 3) (pp. 41-48). Pretoria, South Africa: PME.

Lockwood, E., DeJarnette, A. F., \& Thomas, M. (2019) Computing as a mathematical disciplinary practice. Journal of Mathematical Behavior, 54. doi:10.1016/j.jmathb.2019.01.004

Lockwood, E. \& Erickson, S. (2017). Undergraduate students' initial conceptions of factorials. International Journal of Mathematical Education in Science and Technology, 48(4), 499519. doi:10.1080/0020739X.2016.1259517.

Lockwood, E., \& Gibson, B. (2016). Combinatorial tasks and outcome listing: Examining productive listing among undergraduate students. Educational Studies in Mathematics, 91(2), 247-270. doi:10.1007/s10649-015-9664-5.

Lockwood, E. \& Purdy, B. (2019a). Two undergraduate students' reinvention of the multiplication principle. Journal for Research in Mathematics Education (50)3, 225-267.

Lockwood, E. \& Purdy, B. (2019b) An unexpected outcome: Students' focus on order in the multiplication principle. International Journal of Research in Undergraduate Mathematics Education.

Lockwood, E., Reed, Z., \& Caughman, J. S. (2017). An analysis of statements of the multiplication principle in combinatorics, discrete, and finite mathematics textbooks. International Journal of Research in Undergraduate Mathematics Education, 3(3), 381416. doi:10.1007/s40753-016-0045-y.

Lockwood, E., Swinyard, C. A., \& Caughman, J. S. (2015). Patterns, sets of outcomes, and combinatorial justification: Two students' reinvention of counting formulas. 
International Journal of Research in Undergraduate Mathematics Education, 1(1), 2762. doi:10.1007/s40753-015-0001-2.

Lockwood, E., Wasserman, N. H., \& McGuffey, W. (2018). Classifying combinations: Do students distinguish between different categories of combination problems? International Journal of Research in Undergraduate Mathematics Education, 4(2), 305-322. DOI: 10.1007/s40753-018-0073-X.

Lockwood, E. \& Reed, Z. (2018). Reinforcing mathematical concepts and developing mathematical practices through combinatorial activity. In E. W. Hart, E \& J. Sandefur (Eds.), Teaching and learning of discrete mathematics worldwide: Curriculum and research (93-110). Cham, Switzerland: Springer.

Lockwood, E. \& Reed, Z. (In press). Using an actor-oriented perspective to explore one undergraduate student's repeated reference to a particular problem in a combinatorial context. Submitted to C. Hohensee \& J. Lobato (Eds.) Transfer of learning: Progressive perspectives for mathematics education and related fields.

Lockwood, E., Reed, Z, \& Erickson, S. (In press). Undergraduate students' combinatorial proof of binomial identities. To appear in Journal for Research in Mathematics Education.

Maher, C. A., \& Martino, A. M. (1996a). The development of the idea of mathematical proof: A 5-year case study. Journal for Research in Mathematics Education, 27(2), 194-214.

Maher, C. A., \& Martino, A. M. (1996b). Young children inventing methods of proof: The gang of four. In L. Steffe, P. Nesher, P. Cobb, G. Goldin, \& B. Greer (Eds.), Theories of mathematical learning. Hillsdale, NJ: Erlbaum.

Maher, C. A., Powell, A. B., \& Uptegrove, E. B. (Eds.). (2011). Combinatorics and reasoning: Representing, justifying and building isomorphisms. New York: Springer.

Maher, C. A. \& Speiser, R. (1997). How far can you go with block towers? Journal of Mathematical Behavior, 16(2), 125-132.

Maher, C. A., Sran, M. K., \& Yankelewitz, D. (2011). Making pizzas: reasoning by cases and by recursion. In C. A. Maher, A. B. Powell, \& E. B. Uptegrove (Eds.) Combinatorics and reasoning: Representing, justifying and building isomorphisms (pp. 59-72). New York: Springer.

Maher, C. A. \& Yankelewitz, D. (2011). Representations as tools for building arguments. In C. A. Maher, A. B. Powell, \& E. B. Uptegrove (Eds.) Combinatorics and reasoning: Representing, justifying and building isomorphisms (pp. 17-26). New York: Springer.

Mamona-Downs, J. \& Downs, M. (2004). Realization of techniques in problem solving: the construction of bijections for enumeration tasks. Educational Studies in Mathematics, 56, $235-253$. 
Martin, D.B. (2015). Comments prepared for turning the Common Core into reality in every classroom plenary session. National Council of Teachers of Mathematics 2015 Research Conference: Boston, MA.

Martin, G. E. (2001). The art of enumerative combinatorics. New York: Springer.

Mazur, D. R. (2010). Combinatorics: A guided tour. Washington, DC: MAA.

Miller, D. W. (1991). Discovering and applying Euler's formula. In M. J. Kenney \& C. R. Hirsch (Eds.), Discrete mathematics across the curriculum, K-12: 1991 yearbook (pp. 96-103). Reston, VA: National Council of Teachers of Mathematics.

Muter, E. M., and Maher, C. A. (1998) Recognizing isomorphism and building proof: Revisiting earlier ideas." Proceedings of the Twentieth Annual Meeting of the North American Chapter of the International Group for the Psychology of Mathematics Education, pp. 461-67. Raleigh, N.C.: 1998.

Muter, E. M. \& Uptegrove, E. B. (2011). Representations and connections. In C. A. Maher, A. B. Powell, \& E. B. Uptegrove (Eds.) Combinatorics and reasoning: Representing, justifying and building isomorphisms (pp. 105-120). New York: Springer.

National Council of Teachers of Mathematics. (1980). An agenda for action: Recommendations for school mathematics of the 1980s. Reston, VA: Author.

National Council of Teachers of Mathematics. (1989). Curriculum and evaluation standards for school mathematics. Reston, VA: Author.

National Council of Teachers of Mathematics. (1991). Professional standards for teaching mathematics. Reston, VA: Author.

National Council of Teachers of Mathematics. (2000). Principles and standards for school mathematics. Reston, VA: Author.

National Governors Association Center for Best Practices, \& Council of Chief State School Officers. (2010). Common Core Standards for Mathematics.

Nunes, T. \& Bryant, P. (1996). Children doing mathematics. Oxford, United Kingdom: Blackwell Publishers.

Papert, S. (1990). Mindstorms: Children, computers, and powerful ideas. New York, NY: Perseus.

Pei, C., Weintrop, D., \& Wilensky, U. (2018). Cultivating computational thinking practices and mathematical habits of mind in lattice land. Mathematical Thinking and Learning, 20(1), 75-89, doi:10.1080/10986065.2018.1403543 
Powell, A. B., \& Maher, C. A. (2003). Heuristics of twelfth graders building isomorphisms. In N. A. Pateman, B. J. Dougherty \& J. T. Zilliox (Eds.), Proceedings of the 2003 Joint Meeting of PME and PMENA (Vol. 4, pp. 23-30). Honolulu: CRDG, College of Education, University of Hawai'i.

Powell, A. B. (2011). Block towers: Co-construction of proof. In C. A. Maher, A. B. Powell, \& E. B. Uptegrove (Eds.) Combinatorics and reasoning: Representing, justifying and building isomorphisms (pp. 97-104). New York: Springer.

Reed, Z. \& Lockwood, E. (2018). Generalizing in combinatorics through categorization. In A. Weinberg, C. Rasmussen, J. Rabin, M. Wawro, and S. Brown (Eds.) Proceedings of the $21^{\text {st }}$ Annual Conference on Research in Undergraduate Mathematics Education, (pp. 311-325). San Diego, CA: San Diego State University.

Rosenstein, J. G., Franzblau, D. S., Roberts, F. S. (Eds.). (1997). DIMACS series in discrete mathematics and theoretical computer science: Discrete mathematics in the schools. (Vol. 36). American Mathematical Society and National Council of Teachers of Mathematics.

Schielack, J. F. (1991). Primary experiences in learning what (as well as how) to count. In M. J. Kenney \& C. R. Hirsch (Eds.), Discrete mathematics across the curriculum, K-12: 1991 yearbook (pp. 44-50). Reston, VA: National Council of Teachers of Mathematics.

Sfard, A. (1995). The development of algebra: Confronting historical and psychological perspectives. Journal of Mathematical Behavior, 14, 15-39.

Sinclair, N. \& Patterson, M. (2018). The dynamic geometrisation of computer programming, Mathematical Thinking and Learning, 20(1), 54-74, doi:10.1080/10986065.2018.1403541

Spangler, D. A. (1991). The pigeonhole principle: A counting technique for the middle grades. In M. J. Kenney \& C. R. Hirsch (Eds.), Discrete mathematics across the curriculum, K-12: 1991 yearbook (pp. 55-58). Reston, VA: National Council of the Teachers of Mathematics.

Speiser, R. (2011). Block towers: From concrete objects to conceptual imagination. In C. A. Maher, A. B. Powell, \& E. B. Uptegrove (Eds.) Combinatorics and reasoning: Representing, justifying and building isomorphisms (pp. 73-88). New York: Springer.

Sriraman, B., \& English, L. D. (2004). Combinatorial mathematics: Research into practice. Mathematics Teacher, 98(3), 182-191.

Tarlow, L. D. (2011). Pizzas, towers, and binomials. In C. A. Maher, A. B. Powell, \& E. B. Uptegrove (Eds.) Combinatorics and reasoning: Representing, justifying and building isomorphisms (pp. 121-132). New York: Springer. 
Tarlow, L. D. \& Uptegrove, E. B. (2011). Block towers: Co-construction of proof. In C. A. Maher, A. B. Powell, \& E. B. Uptegrove (Eds.). Combinatorics and reasoning: Representing, justifying and building isomorphisms (pp. 97-104). New York: Springer.

Tillema, E. S. (2011). Students' combinatorial reasoning: The multiplication of binomials. Paper presented for the Thirty-Third Annual Meeting of the International Group for Psychology of Mathematics Education in North America. Reno, Nevada: University of Nevada.

Tillema, E. S. (2013). A power meaning of multiplication: Three eighth graders' solutions of Cartesian product problems. Journal of Mathematical Behavior, 32, 331-352.

Tillema, E. S. (2014). Students' coordination of lower and higher dimensional units in the context of evaluating sums of consecutive whole numbers. Journal of Mathematical Behavior, 36, 51-72.

Tillema, E. S. (2018). An investigation of $6^{\text {th }}$ graders' solutions of combinatorics problems and representation of these problems using arrays. Journal of Mathematical Behavior, 52, 120 .

Tillema, E. S. (in press). Students' solution of arrangement problems and their connection to Cartesian product problems. Mathematical Thinking and Learning.

Tillema, E. S., \& Burch L. J. (2020). Leveraging combinatorial and quantitative reasoning to support the generalization of advanced algebraic identities. Invited paper presentation at the International Congress on Mathematical Education to the Topic Study Group in the Teaching and Learning of Discrete Mathematics in Shanghia, China.

Tillema, E. S. \& Gatza, A. (2016). A quantitative and combinatorial approach to non-linear meanings of multiplication. For the Learning of Mathematics, 36(2), 26-33.

Tillema, E. S. \& Gatza, A.M. (2017). The processes and products of students' generalizing activity. Research report at the Thirty Ninth Annual Meeting of the International Group for Psychology of Mathematics Education in North America, Indianapolis, IN: HAMTE.

Tucker, A. (2002). Applied combinatorics (4th ed.). New York: John Wiley \& Sons.

Vansco, Ö, Beregszaszi, E., Burian, H, Emese, G., Stettner, E., \& Szitanyi, J. (2017). Complex mathematics education in the $21^{\text {st }}$ century: Improving combinatorial thinking based on Tamás Varga's heritage and recent research results. In E. W. Hart \& J. Sandefur (Eds.) Teaching and learning discrete mathematics worldwide: Curriculum and research (111136). Cham, Switzerland: Springer International Publishing.

Wasserman, N. H. \& Galarza, P. (2019). Conceptualizing and justifying sets of outcomes with combination problems. Investigations in Mathematical Learning, 11(2), 83-102. doi:10.1080/19477503.2017.1392208 